\title{
Endovascular Therapy for Abdominal Pregnancy
}

\author{
Sarah Frischhertz, MD, ${ }^{1}$ Jolisha Eubanks-Bradley, MD, ${ }^{2}$ Patrick Gilbert, MD ${ }^{1}$ \\ ${ }^{1}$ Department of Radiology, Ochsner Clinic Foundation, New Orleans, LA ${ }^{2}$ Department of Obstetrics and Gynecology, Ochsner Clinic \\ Foundation, New Orleans, LA
}

\section{INTRODUCTION}

Anomalous placentation carries a high risk of maternal and fetal morbidity and mortality. ${ }^{1-3}$ These placental abnormalities include placenta previa and the spectrum of invasive placenta, including placenta accreta, placenta increta, and placenta percreta. ${ }^{2}$ However, the placentation disorder that perhaps carries the greatest morbidity and mortality to both the mother and fetus is abdominal ectopic pregnancy in which the placenta implants in an extrauterine location. We present a case of such a pregnancy and its management.

\section{HISTORY}

A 34-year-old gravida 2 para 0101 presented as a transfer from an outside hospital at 20 weeks, 5 days' gestation after an ultrasound at the outside hospital showed a left adnexal ectopic pregnancy extending into posterior cul-de-sac and extrauterine placenta (Figure 1). The patient's only prior obstetric history was a 36-week spontaneous vaginal delivery. Per the transferring obstetrician, the patient had had an early ultrasound, copies of which could not be located, that did not identify an ectopic pregnancy. The patient had an abnormal second trimester prenatal screening (quad screen test) that prompted referral to a maternal fetal medicine specialist who diagnosed her with an ectopic abdominal pregnancy. The patient was admitted to the hospital, declined intervention, and was discharged at her request. However, further evaluation and counseling from her primary obstetrician prompted the patient's transfer to our facility.

Upon arrival, the patient was asymptomatic without significant abdominal pain, nausea, or vomiting. Initial abdominal examination revealed no rebound tenderness or guarding, but the pregnancy was palpable left from midline. Repeat ultrasound at our facility confirmed an abdominal pregnancy posterior to the uterus with placenta that originated at the left pelvic side wall extending anteriorly. Blood flow to the placenta was provided by feeding vessels originating from the internal iliac artery and anterior abdominal wall. The fetus measured in the $14^{\text {th }}$ percentile. Amniotic fluid index was normal. Discussion with the patient about the risk of an abdominal pregnancy-including massive hemorrhage and the potential for fetal and maternal morbidity and mortalityresulted in the patient's decision to terminate the pregnancy.

\section{RADIOGRAPHIC APPEARANCE AND TREATMENT}

Computed tomography angiography was performed to better evaluate the placental vascular supply (Figure 2). This study confirmed the extension of the placenta to the anterior abdominal wall with vascular supply to the placenta primarily originating from the left internal iliac artery. Because of these findings, the decision was made to proceed with potassium chloride injection of the fetus, and interventional radiology was consulted for image-guided embolization of the placenta.

Pelvic angiography was performed through a left radial approach. Selection of the internal iliac arteries demonstrated hypertrophied uterine arteries with the left greater than the right, supplying a hypervascular placenta (Figure 3A). A mixture of 500-700 $\mu \mathrm{m}$ and 700-900 $\mu \mathrm{m}$ Embosphere Microspheres (Merit Medical) as well as a large volume of Gelfoam slurry (Pfizer) was injected through a microcatheter to the left uterine artery. A mixture of 300-500 $\mu \mathrm{m}$ and 500-700 $\mu \mathrm{m}$ Embosphere Microspheres and Gelfoam was then delivered to the right uterine artery. Subsequent angiography revealed a pruned tree appearance compatible with significant devascularization of the placenta (Figure 3B). Final pelvic arteriogram showed minimal residual supply to the placenta without aberrant placental arterial supply (Figure 3C). The patient was discharged from the hospital 7 days postembolization with plans for conservative management and serial laboratory evaluation.

Follow-up contrast computed tomography of the pelvis performed 10 weeks postembolization showed the size of the extrauterine gestation was unchanged. However, the soft tissue previously identified as placenta was without enhancement, compatible with complete termination of blood supply to the placenta (Figure 4). Additionally, the prior vascular prominence surrounding the uterus had resolved. At this time, the patient denied pain, nausea, vomiting, or other

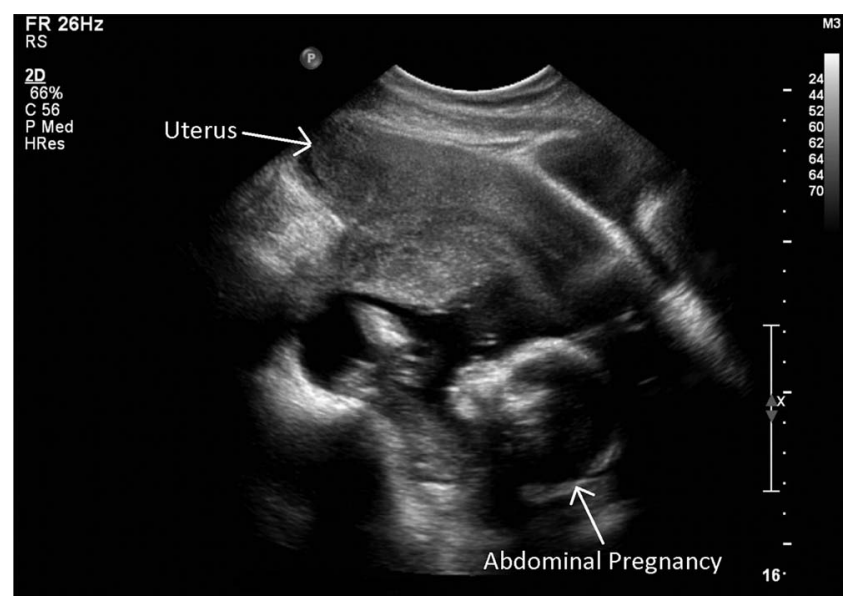

Figure 1. Pelvic ultrasound identified an extrauterine pregnancy. 

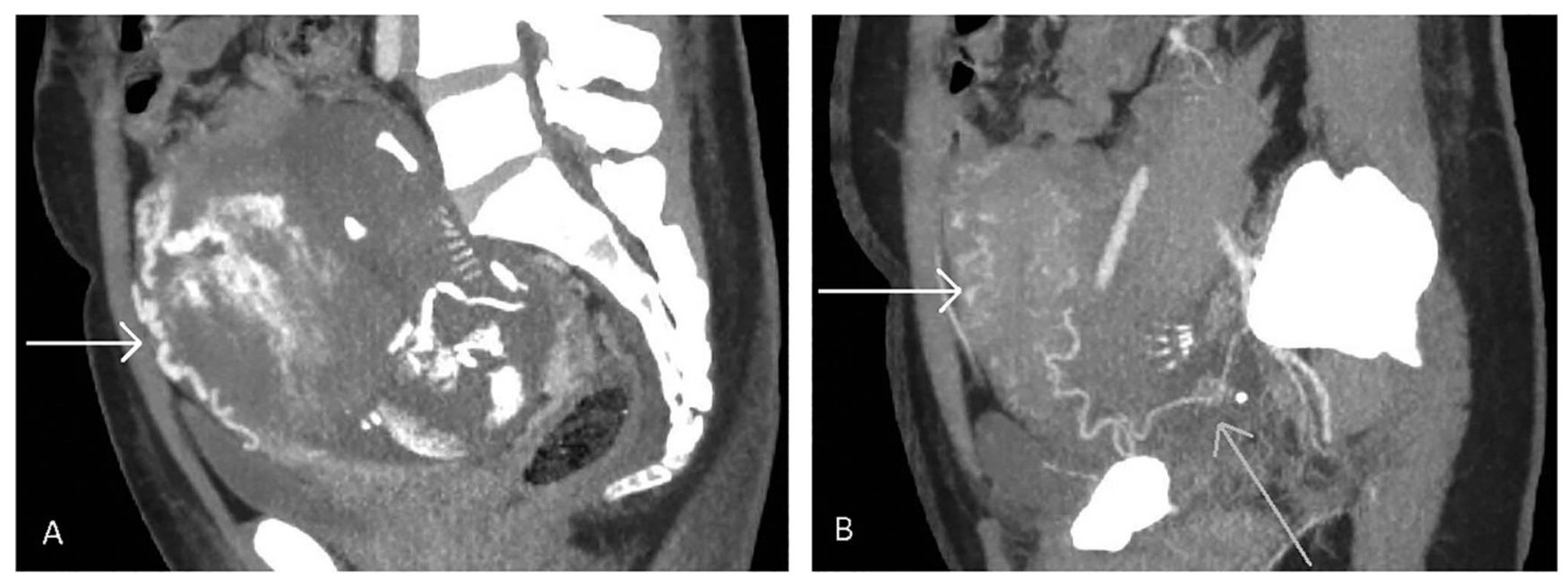

Figure 2. Pelvic computed tomography angiography demonstrated placentation along the anterior abdominal wall (A and $B$, white arrow), with primary placental vascular supply originating from the left internal iliac artery (B, gray arrow).

symptoms. A second placental embolization was planned, but the procedure was deemed not necessary because of the dramatic reduction in placental blood flow. At 13 weeks postembolization, the patient underwent exploratory laparotomy with lysis of adhesions and evacuation of the fetal contents and gestational sac with an estimated operative blood loss of $100 \mathrm{cc}$. The patient did well postoperatively although her course was complicated by elevated blood pressure, a urinary tract infection, and mild nausea and vomiting. She was discharged on postoperative day 7 . When the patient was contacted 6.5 weeks postoperatively, she was without complaints and able to carry on all her regular activities. Because of the significant time required to travel to our facility, she opted to conduct further follow-up with her local primary care physician. Her fertility status is unknown.

\section{DISCUSSION}

To minimize fetal and maternal morbidity and mortality, anomalies of placentation are complications requiring a multidisciplinary approach, including a team of maternal fetal medicine specialists, anesthesiologists, neonatologists, interventional radiologists, and blood bank and nursing
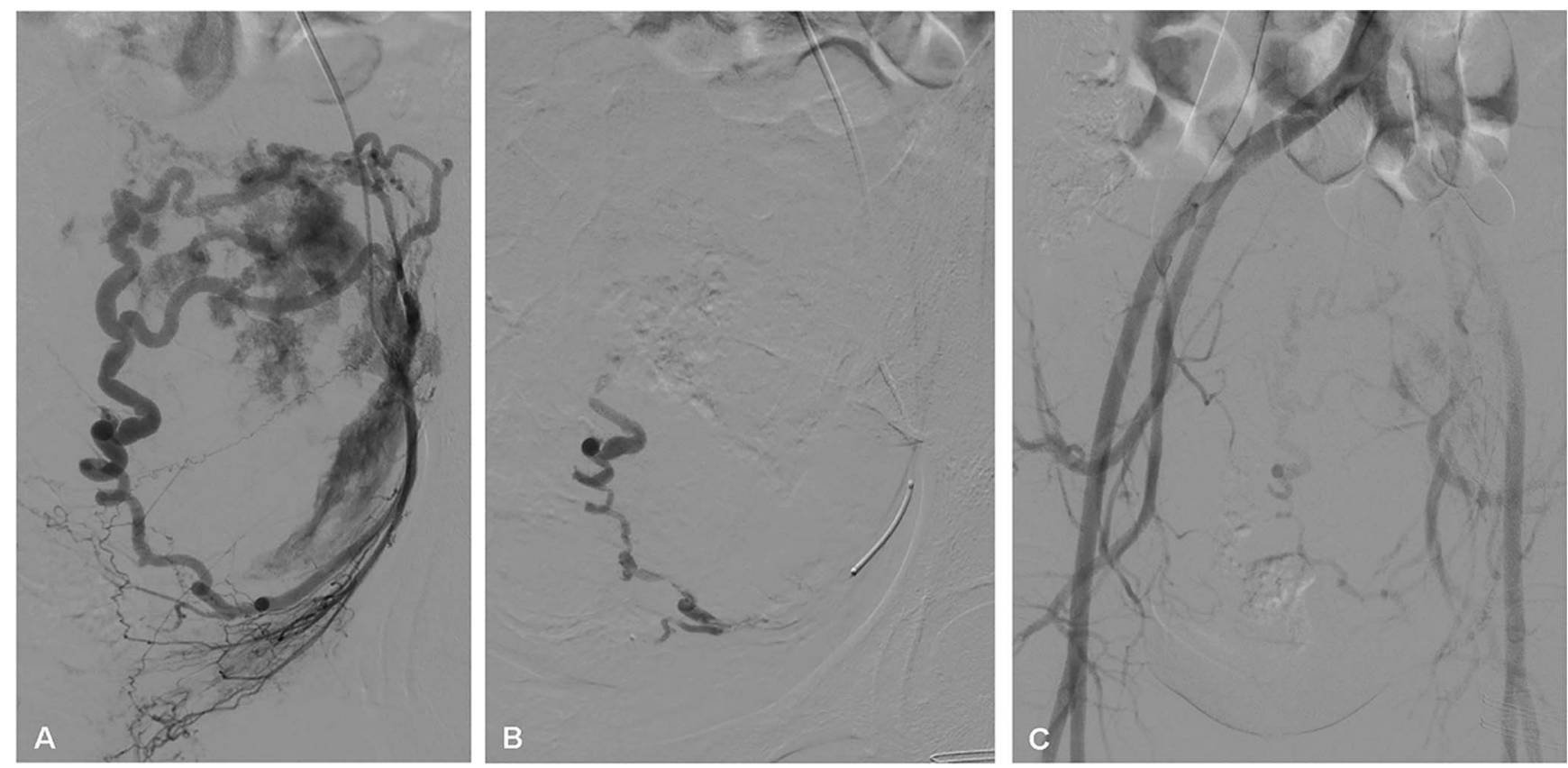

Figure 3. A. Preembolization angiography with selection of the left internal iliac artery demonstrated a hypertrophied uterine artery supplying a hypervascular placenta. B. Postembolization left uterine artery angiography revealed significant placental devascularization. C. Angiography on the right yielded similar results, with the final pelvic arteriogram showing minimal residual supply to the placenta without aberrant placental arterial supply. 

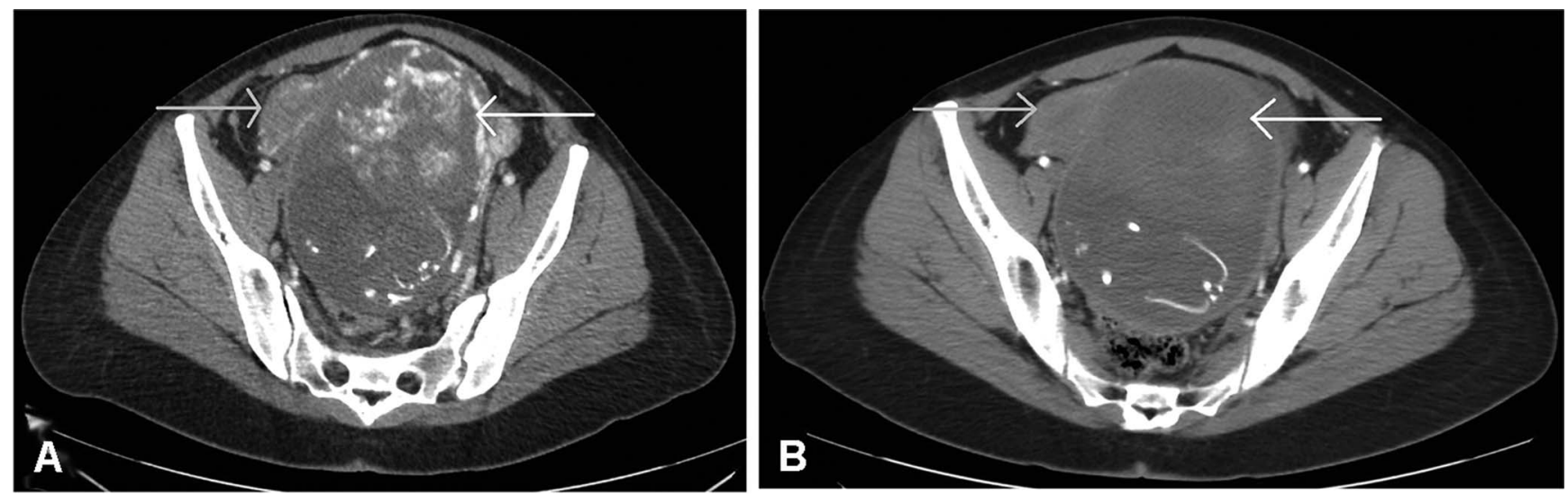

Figure 4. Axial pelvic computed tomography images (A) preembolization and (B) postembolization demonstrated complete resolution in the enhancement of the heterogeneous placental soft tissue within the anterior gestational sac (white arrow) and resolution of the vascular congestion surrounding the uterus (gray arrow).

personnel. In cases of invasive placenta, debate surrounds the question of appropriate management to minimize the risk of peripartum and postpartum hemorrhage. Primary endpoints include maintaining fetal viability, reducing maternal blood loss, and if possible, preserving fertility. ${ }^{1-5}$ At times, the means to achieve each endpoint are in direct conflict. Perhaps no case demonstrates this concept more clearly than an ectopic abdominal pregnancy.

Ectopic abdominal pregnancies are a rare variant in this collection of disorders. While these cases require considerations and treatment similar to other placental anomalies, substantial risk to both the mother and fetus in carrying the pregnancy to term often obviates turning the focus of treatment from preserving fetal viability to protecting maternal health. In our case, fetal compromise was evident on initial imaging through small fetal size. This finding, in conjunction with the inherent high risk of spontaneous maternal exsanguination with an abdominal pregnancy, prohibited the guarantee of a safe continued pregnancy, particularly if the blood supply via the internal iliac artery continued to proliferate.

Through a multidisciplinary approach and image-guided decision making, our case demonstrates the utility of preoperative uterine artery embolization. Uterine artery embolization is well described in the literature as a safe option for reducing maternal morbidity and mortality for treatment of other anomalies of placentation such as placenta previa and the spectrum of invasive placenta. While less common, intraarterial targeted embolization of nontubal ectopic pregnancies has also been described. ${ }^{6,7}$ Similar to our case, a few reports detail cases with successful placental embolization specific to abdominal ectopic pregnancy. ${ }^{8,9}$ Our case contributes to this body of data, with postembolization imaging demonstrating a dramatic decrease in blood flow to the abnormally located placenta. Without a doubt, this decrease in blood flow reduced the otherwise inevitable and significant operative blood loss during fetal evacuation. This experience, in conjunction with previously reported cases, leads us to believe uterine artery embolization is a critical component of management in this complicated and delicate spectrum of disease.

\section{ACKNOWLEDGMENTS}

The authors have no financial or proprietary interest in the subject matter of this article.

\section{REFERENCES}

1. Izbizky C, Meller C, Grasso M, et al. Feasibility and safety of prophylactic uterine artery catheterization and embolization in the management of placenta accrete. J Vasc Interv Radiol. 2015 Feb;26(2):162-169; quiz 170. doi: 10.1016/j.jvir.2014.10.013.

2. Niola R, Giurazza F, Nazzaro G, et al. Uterine artery embolization before delivery to prevent postpartum hemorrhage. J Vasc Interv Radiol. 2016 Mar;27(3):376-382. doi: 10.1016/j.jvir.2015.12.006.

3. Angstmann T, Gard G, Harrington T, Ward E, Thomson A, Giles $W$. Surgical management of placenta accreta: a cohort series and suggested approach. Am J Obstet Gynecol. 2010 Jan;202(1):38.e1-e9. doi: 10.1016/j.ajog.2009.08.037.

4. Diop A, Chabrot P, Bertrand A, et al. Placenta accreta: management with uterine artery embolization 17 cases. J Vasc Interv Radiol. 2010 May;21(5):644-648. doi: 10.1016/j.jvir.2010.01.015.

5. Picel A, Wolford B, Cochran RL, Ramos GA, Roberts AC. Prophylactic internal iliac artery occlusion balloon placement to reduce operative blood loss in patients with invasive placenta. J Vasc Interv Radiol. 2018 Feb;29(2):219-224. doi: 10.1016/j.jvir.2017.08.015.

6. Krissi H, Hiersch L, Stolovitch N, Nitke S, Wiznitzer A, Peled Y. Outcome, complications and future fertility in women treated with uterine artery embolization and methotrexate for non-tubal ectopic pregnancy. Eur J Obstet Gynecol Reprod Biol. 2014 Nov;182:172-176. doi: 10.1016/j.ejogrb.2014.09.026.

7. Fornazari VA, Szejnfeld D, Elito J, Goldman SM. Interventional radiology and endovascular surgery in the treatment of ectopic pregnancies. Einstein. 2015 Jan-Mar;13(1):167-169. doi: 10.1590/S1679-45082015MD3168.

8. Cardosi RJ, Nackley A, Londono J, Hoffman MS. Embolization for advanced abdominal pregnancy with a retained placenta. A case report. J Reprod Med. 2002 Oct;47(10):861-863.

9. Rahaman J, Berkowitz R, Mitty H, Gaddipati S, Brown B, Nezhat F. Minimally invasive management of an advanced abdominal pregnancy. Obstet Gynecol. 2004 May;103(5 Pt 2):1064-1068. doi: 10.1097/01.AOG.0000127946.14387.48. 